\title{
Physical Therapy Japan Vol. 42 (2015) ABSTRACTS
}

*The Japanese Physical Therapy Association publishes the "Journal of the Japanese Physical Therapy Association", the most recent Japanese volume (Physical Therapy Japan) being number 42, which includes 41 articles. Each year 8 issues are published. *For the past 18 years, the English volume of "The Journal of the Japanese Physical Therapy Association" has been published once a year. The journal has changed its' name to "Physical Therapy Research" in 2016.

*To further acquaint our English volume readers with the articles published in the Japanese volume, the English abstracts of the present Japanese volume will be included in the English volume published this year.

\section{Use of Illusion of Motion by the Tendon Vibration to Improve Acute Pain in Patients with Distal Radius Fractures: Early Intervention from the Next Day after Surgery}

\author{
Ryota IMAI, PT \\ Department of Rehabilitation, Kawachi General Hospital \\ Michihiro OSUMI, PT, MS, Shu MORIOKA, PT, PhD \\ Neurorehabilitation Research Center, Kio University \\ Yoshiyuki HIRAKAWA, PT, MHs \\ Department of Rehabilitation, Fukuoka Rehabilitation Hospital \\ Hideki NAKANO, PT, PhD \\ Department of General Rehabilitation, Faculty of Allied Health Science, Yamato University \\ Ryota IMAI, PT, Michihiro OSUMI, PT, MS, Yoshiyuki HIRAKAWA, PT, MHs, \\ Hideki NAKANO, PT, PhD, Shu MORIOKA, PT, PhD \\ Department of Neurorehabilitation, Graduate School of Health Sciences, Kio University \\ Takahiko FUKUMOTO, PT, MS \\ Department of Physical Therapy, Faculty of Health Science, Kio University
}

\begin{abstract}
Purpose: Postoperative acute pain and pain-related anxiety and catastrophizing cause the exacerbation of pain and disability. In this study, we created an illusion of motion through tendon vibration and investigated its effects on sensory and emotional pain and on the range of motion.

Method: As part of a quasi-randomized controlled trial, 14 patients were enrolled. They were assigned to an illusory movement group (7 patients, with vibration of the tendon), or a control group (7 patients, with no vibration). For both the groups, pain (measured by Visual Analog Scale; VAS), pain catastrophizing scale (PCS) score, hospital anxiety and depression scale (HADS) score, and range of motion were assessed before and after the task. Intervention period was 7 days starting from postoperative day 1. Evaluation periods were 7 days, 1 month, and 2 months from the day after surgery. Result: In all the outcomes, we showed main effects and interaction for both the groups (All; $\mathrm{p}<0.05$ ). In period factor, both illusory movement and control group was significantly improvement in the 7 day, 1 month and 2 month from postoperative day compared to the 1 day from postoperative day (p $<0.05$ ).

Discussion: We demonstrated that postoperative pain, range of motion, and emotional pain can be improved by creating an illusion of motion through tendon vibration starting from postoperative day 1 . This clinical study reports an effective measure to prevent the occurrence of chronic pain.
\end{abstract}




\title{
Impact on Orthostatic Hypotension the Day after Gastric Cancer Surgery on the Hospital Course
}

\author{
Kouta YAMAUCHI, PT, Hirofumi SHIMAZOE, PT, Yuya SUZUKI, PT, Kenichi KUMAGAE, PT, \\ Yasuhiro KOYANAGI, PT \\ Department of Rehabilitation, Steel Memorial Yawata Hospital \\ Hiroshi ISHIMURA, MD \\ Department of Vascular Center, Steel Memorial Yawata Hospital \\ Yasuo KAIZUKA, MD \\ Department of Intensive care unit and Emergency, Steel Memorial Yawata Hospital \\ Hidefumi HIGASHI, MD \\ Department of Surgery, Steel Memorial Yawata Hospital
}

Purpose: A key element in enhanced postoperative recovery is early mobilization. However, this may be hindered by orthostatic hypotension $(\mathrm{OH})$. The purpose of the present study was to assess the impact of $\mathrm{OH}$ the day after gastric cancer surgery to the postoperative course.

Methods: A total of 211 patients who underwent elective gastrectomy for gastric cancer with perioperative physiotherapy were examined between 2004 and 2011. The subjects were compared in terms of their hospital course based on presence and absence of $\mathrm{OH}$ the day after surgery.

Results: The time to achieve a sitting position, standing up and walking in the OH-positive group was significantly delayed compared to the $\mathrm{OH}$-negative group. There were no significant differences in the postoperative complications and length of hospital stay between the patients with and without postoperative $\mathrm{OH}$.

Conclusion: $\mathrm{OH}$ on the first day after gastric cancer surgery leads to a delay in mobilization, but the incidence of postoperative complications and length of hospital stay were not significantly different between patients with and without postoperative $\mathrm{OH}$. 


\title{
Estimation of Motor Nerve Function in Community-dwelling Elderly Persons by Analysis of Motor Nerve Conduction Velocity
}

\author{
Ken NISHIHARA, PT, PhD, Takanori KOKUBUN, PT, MS, Fumihiko HOSHI, PT, PhD \\ Department of Physical Therapy, Saitama Prefectural University \\ Hisashi KAWAI, PhD
}

Health Promotion Management Office, Tokyo Metropolitan Geriatric Hospital and Institute of Gerontology

Yu CHIBA, MD

Division of Sensory and Motor System Medicine, University of Tokyo

Toshiaki GOMI, PhD

Faculty of Nursing, Tokyo Ariake University of Medical and Health Sciences

Takanori KOKUBUN, PT, MS

Graduate School of Medical and Dental Sciences, Tokyo Medical and Dental University

Objective: We aimed to evaluate age-related changes of motor nerve function.

Methods: Motor function was investigated by grip strength, timed up and go test (TUG), normal walk, and fastest walk. Motor nerve conduction velocity (CV1) was calculated by measuring latency differences of evoked electromyograms in 25 elderly and 26 young subjects as a conventional method. In addition, motor nerve conduction velocity ( $C V 2)$ from the time at point 0 to the maximum value of the correlation coefficient $\left(R_{\tau} M A X\right)$, and the differences between $C V 1$ and $C V 2(D C V)$ were investigated as the motor nerve conduction velocity-related indexes. Moreover, motor nerve conduction velocity (CV3) was also calculated by measuring time differences between the evoked electromyograms.

Results: The elderly persons were considered to have high levels in the motor function because no difference between the elderly and young subjects was observed in the TUG, and the normal walk test. $C V 1, C V 2$, and $C V 3$ had no significant difference between the two age groups. However, $C V 2$ was lower than CV1 in both the elderly and the young subjects. The possibility of conducting abnormal values of $C V 3$ was shown in certain evoked electromyograms. A negative correlation was observed between $R_{\tau} M A X$ and $D C V$ in the elderly and a positive correlation was observed between $R_{\tau} M A X$ and CV2 in the young.

Conclusions: It was suggested that for the older individuals, including those that maintain a higher level of physical strength and motor nerve conduction velocity, an age-related change of motor nerve function could be estimated with the present methods. 


\title{
Initiation of Early Mobilization to Maintain Walking Ability at Discharge in Heart Failure Patients with Clinical Scenarios 1 and 2
}

\author{
Tetsuya OZAWA, PT, MS, Masakazu SAITOH, PT, PhD, Kentaro HORI, PT \\ Sakakibara Heart Institute
}

Junko SAKAMOTO, PT, MS, Mitsutoshi AKIHO, PT, MS

Mitsui Memorial Hospital

Mariko NAKAZAWA, PT, Masaki HARA, PT

Ayase Heart Hospital

Daisuke OKAMURA, PT

St Luke’s International Hospital

Purpose: To assess the relationship between initiation of early mobilization and walking ability at discharge in heart failure (HF) patients with clinical scenarios (CS) 1 and 2.

Methods: Elderly HF patients (aged $\geq 65$ years) who were hospitalized and received in-hospital phase physiotherapy between June 2011 and June 2013 were eligible to enter the study. Six hundred and seven HF patients with CS1 and 2 (339 males, 268 females, aged $80 \pm 7$ years) were enrolled in the present study. We divided these patients into 2 groups; independent walking group and assisted walking group, according to the walking ability at discharge. We evaluated the difference in progress of cardiac rehabilitation between two groups and the determinants of walking ability at discharge.

Results: Age (odds ratio 1.13, $\mathrm{p}<0.01$ ) and initiation of walking (odds ratio 1.10, $\mathrm{p}<0.05$ ) were related to walking ability at discharge. Using the receiver-operating characteristics curve analysis to predict walking ability at discharge, initiation of walking 4 days after hospital admission was identified as the cut-off value for determining walking ability at discharge (sensitivity 0.689 , specificity 0.520 , area under the curve 0.643 , positive predictive value $28 \%$, negative predictive value $87 \%$, p $<0.01$ ).

Conclusion: Age and initiation of walking were independent determinants of walking ability at discharge in elderly HF patients. Initiation of walking 4 days after hospital admission was identified as the cut-off value for determining walking ability at discharge. 
The Preoperative Factors that Influence the Severity of Dynamic Equinus During Walking after Selective Hip Muscle Release Surgery in Ambulant Cerebral Palsy Patients

\author{
Yasuaki KUSUMOTO, PT, MS \\ Department of Physical Therapy, Faculty of Health Sciences, Tokyo University of Technology \\ Osamu NITTA, PT, PhD \\ Department of Physical Therapy, Faculty of Health Sciences, Tokyo Metropolitan University \\ Tadamitsu MATSUDA, PT, PhD \\ Department of Physical Therapy, Faculty of Health Sciences, Uekusa-Gakuen University \\ Nobumasa NISHINO, PT, Sayaka MATSUO, PT, Kenji TAKAKI, PT, Chisato WAKABAYASHI, PT, \\ Youhei TSUKUI, PT, Haruka HOSHINO, PT \\ Department of Rehabilitation, Minamitama Orthopaedic Hospital
}

Purpose: This study aimed to clarify the preoperative factors that influence the severity of dynamic equinus during walking after selective hip muscle release surgery in ambulant cerebral palsy patients and to determine guidances for physical therapy.

Methods: Seventeen patients (17 limbs) with cerebral palsy of Gross Motor function Classification System (GMFCS) level I or II who underwent selective hip muscle release surgery were selected. We assigned these patients to the reduction, and non-reduction groups on the basis of the change in severity of dynamic equinus during walking at 8 weeks postoperatively. We examined the range of motion, modified Ashworth scale score, and joint torque for the lower extremity.

Results: Compared with the non-reduction group, the reduction group, showed a significantly greater value when Dorsiflexion with Knee Joint Extention was subtracted from Dorsiflexion with Knee Joint Flexion and a significantly lesser value for the popliteal angle at 8 weeks after surgery. The modified Ashworth scale, and joint torques of the lower extremity showed non-significant differences between groups.

Conclusions: The preoperative factors that influenced the change in severity of dynamic equinus during walking after selective hip muscle release surgery in ambulant cerebral palsy patients were extensibility of the soleus and hamstring muscles. These findings suggest that when providing physical therapy for patients with cerebral palsy, the severity change of dynamic equinus during walking can be alleviated after hip surgery by maintaining the extensibility of each muscle. 
Factors Associated with Depressive Symptom in Workers: A Path Analysis Model

Kazuki UEMURA, PT, PhD

Institute of Innovation for Future Society, Nagoya University

Shuhei TAKAHASHI, PT, MS, Yasushi UCHIYAMA, PT, PhD

Department of Physical Therapy, Graduate School of Medicine, Nagoya University

Tsukimi TSUKADA, PHN, MS

Panasonic Corporation Eco Solutions Company

Hiroyasu OGURA, MD

Department of Orthopedics, Kusaka Hospital

Purpose: This study aimed to utilize path analysis to investigate and model the associations between factors associated with depressive symptom in workers.

Methods: Three hundred and forty-six workers (mean age $=41.9$ years) participated in this study. Depressive symptoms were assessed using the Self-Rating Depression Scale (SDS). The factors measured were psychosocial conditions in the workplace, sleep status, physical activity levels during occupational, transport and leisure time, and musculoskeletal pain. The hypothesized relationships between the factors were modeled. The model was then revised and modified utilizing path analysis. Results: The fit of the path analysis model was good $\left(x^{2}=21.0, p=0.14\right.$, GFI $=0.99$, RMSEA $=$ 0.03). The association between SDS and musculoskeletal pain was mediated by job satisfaction and stress. The association between SDS and physical activity during leisure time was mediated by musculoskeletal pain.

Conclusions: Musculoskeletal pain and leisure time physical activity have both a direct and an indirect influence on depressive symptom among workers. These influences are separate from the influence of psychosocial conditions in the workplace. 


\title{
Pelvic Inclination of the Sagittal Plane in the Sitting Position and Related Factors in Patients with Chronic Hemiplegia
}

\author{
Koshiro HARUYAMA, PT \\ National Hospital Organization Higashisaitama National Hospital \\ Michiyuki KAWAKAMI, MD, PhD \\ Keio University School of Medicine
}

Purpose: The purpose of this study was to investigate the characteristics and factors associated with pelvic alignment on the triplane in patients with chronic hemiplegia in the sitting position by determining the standard values of inclination (pelvic inclination of the sagittal plane).

Methods: This study examined 47 patients with hemiplegia. The relationship between the inclination of the loading rate of the buttocks on the paralyzed side, the Brunnstrom recovery stage in the lower limbs, the loading sensation in the buttocks, Pusher syndrome, and the respective basic ability to move, were investigated by measuring the pelvic alignment on the triplane in the rest sitting position. Results: There was no association between hemiplegia and pelvic alignment on the triplane. The mean inclination of sagittal plane on the paralyzed and nonparalyzed sides were both about 10 degrees. There were no significant differences in the mean values. The anterior pelvic inclination group exhibited a better loading rate in the buttocks on the paralyzed side, more loading sensation in the buttocks, and superior standing-up and walking abilities than the posterior pelvic inclination group.

Conclusion: This study suggested that the pelvic inclination angle of patients with hemiplegia in the sitting position is valuable for screening their basic moving ability, buttocks loads and sensory functions. 
Determinants of Activities of Daily Living at Discharge in Elderly Heart Failure Patients

\author{
Masakazu SAITOH, PT, PhD, Kentaro HORI, PT \\ Sakakibara Heart Institute \\ Daisuke OKAMURA, PT \\ St Luke's International Hospital \\ Junko SAKAMOTO, PT, MSc, Hidetoshi SUZUKI, PT, MSc \\ Mitsui Memorial Hospital \\ Mariko NAKAZAWA, PT, Takashi WATANABE, PT \\ Ayase Heart Hospital \\ Tetsuya OZAWA, PT, MSc \\ Odawara Municipal Hospital
}

Purpose: The aim of this study was to examine the factors determining of activities of daily living (ADL) at discharge in elderly heart failure patients.

Methods: Five hundred and eighty five elderly patients aged over 65 years (female 45\%, $75 \pm 12$ years) who were admitted due to acute decompensated heart failure and started physiotherapy during hospitalization were selected and divided into 2 groups according to the preadmission ADL level; ADLindependent group [Barthel index (BI) $\geqq 70$ points] and ADL-dependent group (BI $<70$ points). We analyzed the determinants of ADL at discharge in the two groups.

Results: In the ADL-independent group, the determinants of deterioration of ADL level at discharge were age, hemoglobin, noradrenaline use in acute phase treatment and initiation of walking exercise. In the ADL-dependent group, the determinants of improvement of ADL level at discharge were geriatric nutritional risk index, body mass index and initiation of standing exercise $(\mathrm{p}<0.05)$.

Conclusion: The determining of deterioration of ADL level in elderly heart failure patients who are ADL-independent before admission are age, severity of anemia, use of inotropic agents and time of initiation of walking exercise. On the other hand, the factors determining improvement of ADL level in elderly heart failure patients who are ADL-dependent before admission are nutrition status and time of initiation of standing exercise. 


\section{Effects of a Whole Body Vibration as a Means for Controlling Spasticity in Post-stroke Patients: An F-wave Study}

Kodai MIYARA, PT, Tomohiro UEMA, PT, Takuya HIROKAWA, PT, Tomokazu NOMA, OT, Keiko IKEDA, PT

Kirishima Rehabilitation Center of Kagoshima University Hospital

Shuji MATSUMOTO, MD, PhD, Megumi SHIMODOZONO, MD, PhD, Kazumi KAWAHIRA, MD, PhD

Graduated School of Medical and Dental Sciences, Kagoshima University

Objective: The purpose of this study was to investigate the mechanism of spasticity suppression using the whole body vibration (WBV) in post-stroke patients with lower-limb hemiplegia. F-wave was used to evaluate the excitability of anterior horn cells in the spinal cord.

Methods: Ten post-stroke patients (mean age, $49.3 \pm 13.0$ years) with lower-limb spasticity were enrolled. Each subject sat on a chair with hip joint angles at $90^{\circ}$ of flexion and knee joint angles at $0^{\circ}$ of extension. WBV was applied at $30 \mathrm{~Hz}(4-8 \mathrm{~mm}$ amplitude) for $5 \mathrm{~min}$ on the hamstring and triceps surae muscles. The Modified Ashworth Scale (MAS) as a measure of spasticity, the F-wave parameters (F-wave amplitude, F/M ratio), the active and passive range of motion (A-ROM, P-ROM), and the 10-m walk test as a measure of walking ability were evaluated before and after the WBV intervention.

Results: The MAS score, the A-ROM for ankle dorsiflexion, P-ROM, and walking ability were significantly improved. Moreover, the F-wave amplitude showed a tendency to decrease, and the F/M ratio significantly decreased.

Conclusions: Our results showed reduction of F-wave amplitude and F/M ratio after the WBV intervention, with a corresponding decrease in the MAS score. Suppression of the anterior horn cells by WBV might have led to the suppression of spasticity in this study. Further investigations are necessary to confirm the applicability of the WBV for the treatment of spasticity in post-stroke patients. 


\title{
Analysis of Movements for Wiping the Anus after Defecation in Older Adults
}

\author{
Yuji OSADA, PT, MS, Masako FUCHI, OT, MS \\ Seiai Rehabilitation Hospital \\ Sumiko YAMAMOTO, Eng, PhD \\ Graduate School International University of Health and Welfare \\ Hideyuki NANRI, OT \\ Moji Hospital
}

Purpose: Aiming to identify the most stable way for older adults to wipe the anus after defecation, this study used 3D motion capture analysis to investigate the kinetic and kinematic features of 3 wiping movements.

Methods: Thirteen older adults each performed 3 wiping movements: wiping from back to front with the pelvis tilted laterally (HR1), wiping from back to front with the pelvis tilted anteriorly (HR2), and wiping from front to back while sitting (HR3). Displacement of the center of mass, change in trunk angle, and vertical reaction forces exerted by the feet and buttocks were compared by one-way analysis of variance and multiple comparisons.

Result: Pelvic lateral tilt angle and lateral displacement of the center of mass were significantly increased in HR1. Trunk extension angle was significantly increased in HR2. Displacement of the center of mass and changes in vertical reaction forces were increased only slightly in HR3.

Conclusions: HR3 involves little movement of the trunk and center of mass and requires only upper trunk movement. Of the wiping movements test, HR3 is therefore considered the most stable way for older adults to wipe the anus after defecation. 
Efficacy of a Home-based Exercise Program for Recently Hospitalized Heart Failure Patients

Koji TAKASE, PT, Koji HIGASHINE, PT, Minoru ODA, PT, Makoto MANABE, PT,

Yoshitaka SHIMADA, PT, Riyo OGURA, MD, Takehumi TAKAHASHI, MD,

Yoshikazu HIASA, MD, PhD

Tokushima Red Cross Hospital

Koji TAKASE, PT, Yoshimi MATSUO, PT, PhD

Mukogawa Women's University

Yukio YANAGISAWA, PT, PhD

Tokushima Bunri University

Purpose: To evaluate the efficacy of a home-based exercise program for recently hospitalized heart failure patients.

Methods: We randomized 35 patients with chronic heart failure into an intervention group $(\mathrm{n}=17)$ and control group $(\mathrm{n}=18)$. The intervention group patients were monitored using an activity monitor for 3 months and followed up weekly via telephone interview by a physical therapist to assess walking steps and symptoms. Intervention group patients followed a step chart to increase the number of walking steps gradually to achieve a target number. Health-related quality of life, 6-min walk distance, and 5-m gait speed were evaluated at baseline, 1 month, and 3 months.

Results: In total, $80 \%$ of eligible patients were able to participate in this study, and $71 \%$ of the intervention group patients completed the program. Exercise intervention significantly improved the 6-min walk distance, 5-m gait speed, and health-related quality of life. No adverse events were observed in either group.

Conclusion: A home-based exercise program monitored by a physical therapist is useful for recently hospitalized heart failure patients. 


\title{
Electromyographic Analysis of a New Lumbar Multifidus Exercise
}

\author{
Masanobu MURAO, PT, Masaaki NAKAJIMA, PT, PhD \\ Graduate School of Health Science, Kibi International University \\ Yoshinobu SATO, PT \\ Chiba Qiball Clinic, Medical Corporation Jinseikai
}

Purpose: To demonstrate the muscle activity characteristic of the new exercise (N-ex) that we devised on the concept of selective contraction lumbar multifidus muscle (LM).

Methods: Twenty-one healthy college students performed three lumbar stabilization exercises: "Abdominal-drawing", "Bird-dog”, and "N-ex". Surface electrodes were used to record the electromyographic signal amplitude of the LM and iliocostalis thoracis muscle (ICLT). Average amplitudes obtained during the exercises were normalized to the amplitude in maximal voluntary contraction (\% MVIC). We compared the three groups' \% MVIC and the Local/Global muscle activity ratio $(\mathrm{L} / \mathrm{G}$ ratio).

Result: N-ex showed a high \% MVIC of the LM significantly to Abdominal-drawing, but no significant difference was observed between the Bird-dog. In addition, N-ex showed the highest L/G ratio significantly of all exercises.

Conclusions: By EMG analysis study, it is clear that N-ex can enable to selective contraction of LM, while maintaining sufficient activity percentage of LM. 
The Effect of the Training of Standing on Single Leg with Eyes Closed

Maiko KANDA, PT, Ryosaku KOBAYASHI, PT, PhD

Geriatric Health Services Facilities Toyouraaikoen

Purpose: The purpose of this study was to clarify whether the standing-on-single-leg time with closed eyes changes in people who declined the time when they train to stand on single leg with their eyes closed.

Methods: The subjects were 20 healthy females for $\leq 40$ seconds the standing on single leg time with eyes closed among the 40 subjects. On physical examination, height, weight, single-leg standing time with eyes open and closed, pressure strength of the toes, plantar 2-point discrimination, and leg power were assessed. We performed a pre-test to determine the maximum duration that subjects were able to standing on single leg (right and left legs) with eyes closed. The leg that remained suspended for a longer duration was termed as the non-training leg, whereas the other leg was termed as the training leg. The subjects' training consisted of standing on single leg, using the training leg with eyes closed, for 120 seconds. The subjects performed these tasks 3 times daily, 3 times per week for 3 weeks. All subjects were post-tested using the exact same procedure after 3 weeks, 3 months and 6 months.

Results: The results revealed that both training and non-training legs showed significant increases in one-leg standing time after the intervention. However the changes in one-leg standing time were significantly greater in the training legs. The training effect was maintained 3-6 months after the end of the assessment. The rest of the variables did not differ significantly.

Conclusions: The 3-week single-leg standing training period had a positive effect on performance in young healthy subjects. 
Modulation of Cortical Excitability by Transcranial Direct Current Stimulation

Shota MIYAGUCHI, PT, MS, Sho KOJIMA, PT, MS

Graduate School of Niigata University of Health and Welfare

Shota MIYAGUCHI, PT, MS, Hideaki ONISHI, PT, PhD, Sho KOJIMA, PT, MS,

Kazuhiro SUGAWARA, PT, PhD, Hikari KIRIMOTO, OT, PhD, Hiroyuki TAMAKI, PhD,

Noriaki YAMAMOTO, MD, PhD

Institute for Human Movement and Medical Sciences, Niigata University of Health and Welfare

Shota MIYAGUCHI, PT, MS

Nakajo Central Hospital

Sho KOJIMA, PT, MS

Tokyo Bay Rehabilitation Hospital

Noriaki YAMAMOTO, MD, PhD

Niigata Rehabilitation Hospital

Purpose: In the present study, we investigated whether anodal transcranial direct current stimulation (tDCS) applied to the primary motor cortex (M1) is affected differential of current intensity or electrode position.

Methods: Nine healthy subjects participated in this study. tDCS was delivered using a pair of salinesoaked surface sponge electrodes for $10 \mathrm{~min}$. The anodal electrode was placed on the scalp above the left M1. The cathodal electrode was placed over the orbit or on the scalp above the right M1 (unilateral tDCS and bilateral tDCS, respectively). All subjects underwent the following four interventions: unilateral tDCS delivered with $1.0 \mathrm{~mA}$ and $2.0 \mathrm{~mA}$ (unilateral_1.0 mA condition and unilateral_2.0 mA condition, respectively) and bilateral tDCS delivered with $1.0 \mathrm{~mA}$ and $2.0 \mathrm{~mA}$ (bilateral_1.0 mA condition and bilateral_2.0 mA condition, respectively). Motor evoked potentials (MEPs) were recorded from the right first dorsal interosseous before and 2 min and 10 min after the intervention.

Results: Under the bilateral_2.0 mA condition, the MEP amplitudes at 2 min and 10 min after the intervention were significantly increased compared with those before the intervention. However, under the other three conditions, the MEP amplitudes remained unchanged.

Conclusion: These results demonstrate that anodal tDCS is highly affected differential of current intensity or electrode position.

Key Words: transcranial direct current stimulation, electrode position, current intensity 


\title{
Effects of Low Intensity and Home-based Pulmonary Rehabilitation on Physical Activity in Elderly Patients with COPD
}

\author{
Atsuyoshi KAWAGOSHI, PT, PhD, Noritaka KIYOKAWA, PT, Keiyu SUGAWARA, PT, PhD, \\ Hitomi TAKAHASHI, PT, PhD \\ Akita City Hospital \\ Shunichi SAKATA, PhD
}

Kumamoto Technology and Industry Foundations

Masahiro SATAKE, PT, PhD, Takanobu SHIOYA, MD, PhD

Akita University Graduate School of Health Sciences

Purpose: We evaluated the effect of low-intensity and home-based pulmonary rehabilitation (PR) on physical activity (PA) in stable elderly patients with chronic obstructive pulmonary disease (COPD).

Methods: We assessed the patients' PA using a newly developed triaxial accelerometer (A-MES ${ }^{\mathrm{TM}}$ [Activity Monitoring and Evaluation System], Solid Brains, Kumamoto, Japan), which measures the time spent in walking, standing, sitting and lying down. The participants were 15 elderly COPD patients (ages $76 \pm 8$ yrs; $\mathrm{FEV}_{1} 56.6 \pm 18.7 \%$ pred). The patients' PA and pulmonary function, exercise capacity (6min walking distance; 6MWD), quadriceps muscle force (QF), BODE (body-mass index, airflow Obstruction, Dyspnea, and Exercise) score and health-related quality of life as measured by the Chronic Respiratory Questionnaire were evaluated before the start of the PR as the baseline and at $1 \mathrm{yr}$ after the completion of the PR. We compared the patients' pre- and post-PR PA and the other parameters listed above and analyzed the relationships between the changes in the time spent in walking and those of the other parameters.

Results: The patients' walking time increased (pre PR: $123 \pm 58$ vs. post PR: $171 \pm 82 \mathrm{~min} /$ day) significantly and their time spent lying down decreased (pre PR: $117 \pm 63$ vs. post PR: $80 \pm 42 \mathrm{~min} /$ day) significantly after the PR. The improvement rate of daily walking time after the PR was significantly correlated with those of BODE score, 6MWD and QF.

Conclusions: These results suggest that low-intensity and home-based PR was effective in improving $\mathrm{PA}$, and the improvements of physiological factors were correlated with increased walking time in stable elderly patients with COPD.

Key Words: Physical Activity, Chronic Obstructive Pulmonary Disease, Low Intensity Exercise, Homebased Pulmonary Rehabilitation 


\title{
Kinetic and Electromyographic Analyses of Sit-to-Stand Motion in Patients with Proximal Femoral Fracture: Comparison of Patients with Pain and without Pain
}

\author{
Takahiro UENO, PT, MS, Koji TAKAHASHI, PT, Takuya ZAMA, PT, Eiji SUZUKI, MD, Dr \\ Saitama Memorial Hospital Rehabilitation Center \\ Takahiro UENO, PT, MS \\ Koshigaya Rehabilitation Care Center \\ Kazuhiko HARA, PT, PhD \\ Graduate School of Saitama Publican University
}

Objective: The purpose of this study is to make kinetic and electromyographic analyses on sit-tostand motion of patients with proximal femoral fracture and to reveal differences in motion patterns and muscular activities during motion according to presence or absence of pain on motion.

Method: Twenty one patients with proximal femoral fracture who had maintained cognitive function and had no serious past medical history were included in the study. Sit-to-stand motion without upper limb support from a chair as high as $120 \%$ of the length of lower limbs was studied using 3D motion analysis system and surface electromyogram. Subjects were classified into groups with and without pain according to presence or absence of pain during motion, and results of motion analyses were compared between the groups.

Result: Pain was detected mainly in quadriceps muscle. Range of hip motion and hip extension moment were significantly small in patients with pain. Knee extension moment was significantly large in patients with pain. The ratio of muscular activities for the affected versus unaffected sides was significantly large in vastus lateralis muscle of patients with pain.

Conclusion: Comprehension by knee joint function for decreased hip joint function was observed in patients experiencing pain during motion, suggesting involvement of compensatory movement in muscular pain development during the recovery phase.

Key Words: Proximal Femoral Fracture, Pain, Sit-To-Stand Motion 


\title{
Examination of Related Factors and Cut-off Values for Determining Walking Independence of Inpatients with Medical Disease in Acute Care Hospitals
}

\author{
Shinya YAMAUCHI, PT, Shinichiro MORISHITA, PT, PhD, Yosuke HONDA, PT, \\ Masahiro NAGASE, PT, Koujiro TSUDA, PT, Satoshi MABUCHI, PT, \\ Norihiko KODAMA, MD \\ Hyogo College of Medicine Hospital \\ Kazuhisa DOMEN, MD \\ Hyogo College of Medicine
}

Purpose: The purpose of this study was to examine related factors and cut-off values for determining walking independence in inpatients with medical disease in acute care hospitals.

Methods: A total of 170 inpatients with medical disease were enrolled in this cross-sectional study. The patients were divided into a walking independent group or a walking dependent group, and outcome measures for determining walking independence were examined by performing logistic regression analysis for factors such as cognitive function, physical function, blood test data, and so on. In addition, cut-off values were calculated from the receiver operating characteristic curve.

Results: The outcome measures and cut-off values of the following four parameters had been extracted by performing logistic regression analysis: knee extension strength, one leg standing retention time, mini-mental state examination (MMSE) score, and total protein (TP) value. In addition, the cut-off value of each parameter was as follows: knee extension strength, $0.30 \mathrm{kgf} / \mathrm{kg}$; one leg standing retention time, $2.93 \mathrm{~s}$; MMSE score, 27/28 points, and TP value, $5.95 \mathrm{~g} / \mathrm{dL}$.

Conclusion: The results of this study revealed that the knee extension strength, one leg standing retention time, MMSE score, and TP value were related to walking independence in inpatients with medical disease in acute care hospitals. It was indicated that these outcome measures were important for determining walking independence.

Key Words: walking independence, related factors, cut-off values 


\title{
Postoperative Changes of Activity Limitation and Participation Restriction within a Six-month Period Following Knee Joint Arthroplasty
}

\author{
Mutsumi NAKAMURA, PT, PhD, Chiyoko KISE, PT, Saho YAMAGATA, PT, Masumi MORITA, PT, \\ Kyoichi HASEGAWA, PT \\ Akabane Central General Hospital \\ Mutsumi NAKAMURA, PT, PhD, Yasutsugu ASAKAWA, PT, PhD, Masahumi MIZUKAMI, PT, PhD \\ Ibaraki Prefectural University of Health Sciences
}

Purpose: The purpose of this study was to investigate the changes of activity limitation and participation restriction of patients within a six-month period following knee joint arthroplasty. Fifty-one knee arthroplasty patients completed the assessment at the following times: pre-surgery, upon discharge (average of three weeks), three months post-surgery and six months post-surgery.

Methods: Subjects were evaluated using the qualifiers'scale of the ICF Core Set in the Activity and Participation component for osteoarthritis by completing the Ministry of Health, Labour and Welfare's "Standard for the Activity and Participation (draft)" with an additional set of specifications created by our team.

Results: The ICF core set data comparison shows significant improvement in all categories assessed from pre-surgery to post-surgery (six months). However, the rate of improvement in participation restriction is more gradual than that in activity limitation.

Conclusions: The improvement of one's real life ability to effectively carry out each activity is slower than that of one's initial ability. The results of the study will help enhance the physical therapeutic understanding of improving the activity and participation abilities of the patients who have had knee joint arthroplasty.

Key Words: Total Knee Arthroplasty, ICF, Activity and Participation 


\section{Quantitative Evaluation of Human Muscle Hardness Using a Newly Developed Ultrasonography}

Eiji FUJITA, PhD, Tetsuo FUKUNAGA, PhD

National Institute of Fitness and Sports in Kanoya

Siho SAWAI, PhD

Japan Women's College of Physical Education

Hisashi TANAKA, BS

Global Health Co. Ltd.

Purpose: The purpose of the present study was to investigate the validity and reliability of a newly developed ultrasonographic method to quantify the hardness of human skeletal muscle. The newly ultrasonographic equipment (muscle hardness ultrasonography; mhULT) consists of an electric pressure gauge built onto an ultrasonographic probe and a portable B-mode ultrasonography device.

Methods: To estimate the validity and reliability of mhULT, three experiments were conducted. In experiment 1 , three phantoms of different hardness were used to estimate the validity and reliability of mhULT. In experiment 2, right elbow flexor muscle hardness was measured at rest twice within a 3-day interval using mhULT. In experiment 3, muscle hardness was measured at rest and during flexion at $15 \%, 30 \%$, and $45 \%$ of maximal voluntary isometric contraction (MVC) to clarify the relationship between muscle hardness and isometric elbow flexion force.

Results: A significant positive correlation was found between the phantom hardness and the hardness measured by mhULT $(r=0.990)$, the stable reproducibility of values measured by mhUT was obtained from an intraclass correlation coefficient of 0.703 to 0.968 (experiment 1). An intraclass correlation coefficient of 0.806 showed a high reproducibility of the measured values. Moreover, a significant positive correlation was found between muscle hardness and muscular force exerted from rest to 45\% MVC ( $\mathrm{r}=$ 0.967) (experiment 3).

Conclusion: These results indicate that the newly developed ultrasonography can accurately and conveniently quantify the hardness of human skeletal muscle.

Key Words: ultrasonography, elbow flexor muscle, muscle hardness 


\title{
Correlation between Clinical Evaluation with Shoulder 36 and Actual Functional Measurement in Shoulder Diseases
}

\author{
Tadashi NAKANO, PT, MS \\ Department of Rehabilitation Sciences, Kansai University of Welfare Sciences
}

Hisayoshi MURANISHI, PT, MS

Department of Physical Therapy, Faculty of Rehabilitation, School of Rehabilitation, Osaka Kawasaki Rehabilitation University

Goya SHINBASHI, PT, Masaki KUWANO, PT, Tamotsu NAKATSUJI, MD, PhD

Department of Rehabilitation, Tsuji-Geka Rehabilitation Hospital

Yoich ITO, MD, PhD

Department of Orthopaedic Surgery, Osaka City University Graduate School of Medicine

Background: The shoulder evaluation sheet Shoulder 36 (Sh36) was recently introduced as a new clinical evaluation tool for shoulder diseases. Sh36 is an assessment sheet based on patients' subjectivity. It consists of 36 questions, including several domains. However, the correlation between the results of clinical evaluation with Sh36 and actual functional measurements is not clear.

Purpose: The purpose of this study was to investigate the aforementioned correlation and assess the clinical significance of Sh36.

Methods: We evaluated 116 shoulders in 116 consecutive patients who had arthroscopic shoulder surgeries. The mean age at surgery was 63.4 years. The 116 shoulders were divided into 3 disease groups as follows: 55 cases of rotator cuff tear, 41 cases of subacromial impingement syndrome, and 20 cases of stiff shoulders. Preoperative clinical evaluation was performed by using Sh36 and obtaining actual functional measurements in three domains such as pain, range of motion, and muscle strength. Correlations between Sh36 and the actual functional measurements in the three domains in the three disease groups were statistically evaluated.

Results: Strong correlations between the three Sh36 domains and the actual functional measurements were observed in the cases of rotator cuff tear and subacromial impingement syndrome. Weak correlations between range of motion and the actual functional measurements were observed in the cases of stiff shoulder.

Conclusions: Significant correlations between the results of the clinical evaluation with Sh36 and the actual functional measurements were observed especially in the cases of rotator cuff tear and subacromial impingement syndrome. Sh36 was demonstrated as a relatively useful clinical evaluation tool for painful shoulders in middle- and old-aged patients.

Key Words: Shoulder36, subjective evaluation, shoulder diseases 


\title{
Clinical Prediction Rules for Ambulation with Incomplete Spinal Cord Injury in Sub-acute Hospital: A Retrospective Study
}

\author{
Kazunori KOSEKI, PT, MS, Kenichi YOSHIKAWA, PT, MS, Takayuki MAEZAWA, PT \\ Ibaraki Prefectural University of Health Sciences Hospital
}

Yasutsugu ASAKAWA, PT, PhD, Masafumi MIZUKAMI, PT, PhD

Ibaraki Prefectural University of Health Sciences

Purpose: We aimed to identify predictors of independent walking in patients with motor incomplete Spinal Cord Injury (miSCI) admitted to a rehabilitation hospital and to thus make a prediction rule.

Method: The subjects were 87 patients with miSCI who, less than 3 months after their injuries, had been admitted to Ibaraki Prefectural University of Health Sciences Hospital between March 2001 and December 2012. Data on demographics and neurological and functional status, including age, sex, cause of injury, American Spinal Injury Association Impairment Scale (AIS) score, tetra- or paraparesis, rolling ability, ability to sit up, sitting ability, ability to stand up, standing ability, Walking Index for Spinal Cord Injury II (WISCI) score, Functional Independence Measure (FIM) motor sub-score, FIM cognitive subscore, and bladder function, were collected retrospectively. A clinical prediction rule was determined by using these scores and a multivariate logistic regression model. Primary outcome measures were independent walking indoors and outdoors, as determined by the Spinal Cord Independence Measure III score at discharge.

Result: After logistic regression analysis, AIS, rolling ability, WISCI, and the FIM cognitive sub-score were selected as predictors of independent walking indoors. In addition, age and standing ability were selected as predictors of independent walking outdoors. We used the two prediction models to create an algorithm for walking independently.

Conclusion: Formulating a prediction rule for independent walking indoors and outdoors in patients with miSCI upon admission to rehabilitation hospitals is very important. This rule can be used to set rehabilitation goals and programs.

Key Words: Spinal Cord Injury, Prognosis Prediction, Ambulation 
Effect of the Spinal Deformity on Postural Control During Quiet Standing in Patients with Adolescent Idiopathic Scoliosis with a Single Curve

\author{
Yukako MIMORI, PT, MS \\ Keio University Hospital \\ Kota WATANABE, MD, PhD, Yohei OTAKA, MD, Toshiyuki FUJIWARA, MD, PhD, \\ Naobumi HOSOGANE, MD, PhD, Morio MATSUMOTO, MD, PhD, Meigen LIU, MD, PhD \\ Keio University School of Medicine \\ Osamu NITTA, PT, PhD \\ Tokyo Metropolitan University \\ Kimitaka HASE, MD, PhD \\ Kansai Medical University
}

Purpose: The purpose of this study was to examine the effect of the location of spinal deformity on postural control during quiet standing in adolescent idiopathic scoliosis (AIS) by comparing the parameters of center of pressure (COP) between patients mainly with thoracic deformity (thoracic group) and those with lumbar deformity (lumbar group).

Methods: This study included 100 patients with AIS (67 in the thoracic group and 33 in the lumbar group, mean age: $18.1 \pm 5.5$ years). We evaluated postural sway by measuring COP during quiet standing, and compared the indices of COP between the two groups. In each group, we also examined the relations of postural sway with age and radiographic parameters.

Results: There was no significant difference in the COP parameters of sway between the two groups. However, the COP parameters of sway correlated with age in the thoracic group. In the lumbar group, the parameters of sway with eyes closed correlated with the Cobb angle. The effect of visual deprivation on length and area of COP was smaller in the lumbar group compared with the thoracic group. In both groups, there was a correlation between the lateral displacement of COP and coronal balance.

Conclusion: The postural sway showed some different characteristics according to the location of spinal deformity. In the lumbar group, the postural sway was affected by the degree of spinal deformity.

Key Words: Spinal deformity, Postural control, Center of pressure 


\title{
Muscle Activation Amplitude and Onset Time in Patients with Chronic Nonspecific Low Back Pain During Trunk Rotation
}

\author{
Masashi TANIGUCHI, PT, MSc \\ Shiga University of Medical Science Hospital \\ Hiroshige TATEUCHI, PT, PhD, Noriaki ICHIHASHI, PT, PhD \\ Graduate School of Medicine, Kyoto University \\ Natsuko NARITA, PT, MSc \\ Independent member
}

Purpose: The objective of the present study was to investigate differences in electromyography (EMG)-recorded amplitude and onset time between individuals with and without chronic nonspecific low back pain (LBP) during trunk rotation.

Methods: Fifteen patients with LBP (9 men and 6 women; $22.5 \pm 2.4$ years) and 15 healthy control subjects ( 9 men and 6 women; $25.2 \pm 5.5$ years) participated in this study. Each subject was instructed to perform trunk rotation as fast as possible after a LED indicator signal. The amplitude and onset time in the onset phase of trunk rotation were recorded using surface EMG and a three-dimensional analysis system from three pairs of abdominal muscles and five pairs of back muscles: the rectus abdominis, internal oblique, external oblique, erector spinae, multifidus, gluteus maximus, and upper/lower fibers of the latissimus dorsi. The Mann-Whitney test was used to determine differences between the two groups.

Results: Patients with LBP showed significantly increased amplitude and delayed onset time in the contralateral external oblique compared with healthy subjects.

Conclusions: These results suggest that chronic nonspecific LBP patients have abnormal muscle activation in the external oblique during trunk rotation.

Key Words: Chronic low back pain, Trunk rotation, Electromyography 


\title{
The Effect of the Direction of the Force at the Ankle Joint on the Shearing Force at the Knee Joint: A Mathematical Simulation Study
}

\author{
Takuma INAI, PT, MSc, Mutsuaki EDAMA, PT, MSc, Tomoya TAKABAYASHI, PT, MSc \\ Graduate School of Niigata University of Health and Welfare \\ Takuma INAI, PT, MSc, Mutsuaki EDAMA, PT, MSc, Tomoya TAKABAYASHI, PT, MSc, \\ Masayoshi KUBO, PT, ScD \\ Institute for Human Movement and Medical Sciences, Niigata University of Health and Welfare \\ Yuta TOKUNAGA, PT, MSc \\ Niigata Rehabilitation Hospital
}

Purpose: The purpose of this study was to examine the direction of the force at the ankle joint that reduces the anterior shearing force at the knee joint during muscle training of the vastus using a mathematical simulation.

Methods: The functional effective muscle combined with the Hill model was used to estimate the muscle forces at the knee joint. The muscle lines of action were determined using the straight line model and the wrapping surface. Behavior of the shearing force at the knee joint was simulated by systematic manipulation of the direction of the force at the ankle joint.

Result: When the direction of the force at the ankle joint changed, bordering on the line connecting the hip joint and the ankle joint, the anterior shearing force at the knee joint also changed.

Conclusion: The findings of this study regarding the direction of the force at the ankle joint may provide the information needed to implement a safe muscle training method for anterior cruciate ligament injuries.

Key Words: Shearing force, Knee joint, Direction of force, Ankle joint, Mathematical simulation 


\title{
Sole Radius of Ankle-Foot Orthoses (AFOs) with an Immobile Ankle Joint for Optimal Energy Conservation during Walking in Young Adults
}

\author{
Yuki MATSUNAGA, PT, MSc \\ Showa University Koto Toyosu Hospital \\ Daisuke NAKAMURA, PT, PhD, Noboru SEKIYA, PT, PhD \\ Showa University
}

Background and purpose: Circular sole of Ankle-Foot Orthoses (AFOs) with an immobile ankle joint has been shown to be effective for ensuring smooth walking regardless of walking speed, shoe height, and load carried. Energy efficiency was reported to be optimal when the circular sole radius was approximately $15 \%$ of subject's body height (BH) (Adamczyk, 2006). However, AFOs with such a radius are assumed to be unstable while standing and walking in daily life. Therefore, the purpose of this study was to clarify energetically optimal circular sole radius of AFOs with a fixed ankle joint in a range of radii.

Method: Twelve male volunteers [mean age of 24.2 years ( $\mathrm{SD}=5.4$ years)] participated in the study. They walked on a treadmill at a speed of $4.5 \mathrm{~km} / \mathrm{h}$ for $7 \mathrm{~min}$ in each footwear condition. The footwear conditions were as follows: sneakers and AFOs with circular sole radius of $15 \%, 21 \%, 27 \%, 33 \%$, $39 \%$, or $45 \% \mathrm{BH}$. We measured the energy consumption rate during the last 1 min of the 7 -min walk.

Result: The energy consumption rate was minimum at a circular sole radius of $27 \% \mathrm{BH}$, and tended to increase with an increase in cadence. The energy consumption rate during walking with AFOs with a circular radius of $27 \% \mathrm{BH}$ was not larger than that with sneakers.

Conclusion: The energetically optimal circular sole radius of AFOs with an immobile ankle joint was $27 \% \mathrm{BH}$, which was larger than the value reported by Adamczyk (2006). Furthermore, the energy consumption with AFOs with a circular sole radius of $27 \% \mathrm{BH}$ was similar to that with sneakers.

Key Words: Walking, Energy efficiency, Ankle fixation, Sole shape, Ankle-foot orthosis 


\title{
Factors Affecting the Perceived Leg Length Discrepancy after Total Hip Arthroplasty: Using a Hierarchical Multiple Regression Analysis
}

\author{
Yuji KAWABATA, PT, Keita GOTO, PT, Satoshi TAKECHI, PT, Mitsuhiro KIMURA, MD \\ Shuto General Hospital, JA Yamaguchi Prefectural Welfare Federation of Agricultural Cooperative
}

Purpose: The purpose of this study was to clarify the factors affecting the perceived leg length discrepancy after total hip arthroplasty.

Methods: The subjects were 67 patients who underwent primary unilateral total hip arthroplasty. We recorded age, gender, and body mass index as basic characteristics. We also measured radiographic leg length discrepancy, Crowe's classification, lateral pelvic tilt angle, and Japanese Orthopedic Association score preoperatively, and radiographic leg length discrepancy, lateral pelvic tilt angle, the amount of leg lengthening, length of offset, pain, hip extension angle, hip adduction angle, hip abduction angle, hip abductor strength, and perceived leg length discrepancy postoperatively. First, the correlation between the parameters and the perceived leg length discrepancy was verified using Spearman's correlation analysis. Subsequently, the parameters showing significant correlation with the perceived leg length discrepancy were used as independent variables in a hierarchical multiple regression analysis, with perceived leg length discrepancy as the dependent variable.

Results: A hierarchical multiple regression analysis revealed that the operative hip adduction angle and postoperative lateral pelvic tilt angle were significantly related to the perceived leg length discrepancy and these relationships were independent of the actual postoperative radiographic leg length discrepancy (coefficient of determination, 0.619).

Conclusion: The results suggest that an increase in the operative hip adduction angle and correction of lateral pelvic tilt are important for improving perceived leg length discrepancy.

Key Words: Total hip arthroplasty, Perceived leg length discrepancy, Hip adduction range of motion 


\title{
Effect of Preoperative Sarcopenia on Postoperative Course in Gastrointestinal Cancer Patients, with Specific Focus on Postoperative Complications, Physical Function Changes, and Quality of Life
}

\author{
Tsuyoshi HARA, PT, PhD, Shusuke KUSANO, MD, PhD \\ International University of Health and Welfare Mita Hospital \\ Akira KUBO, PT, PhD \\ International University of Health and Welfare
}

Purpose: The purpose of this study was to observe the effects of preoperative sarcopenia on the incidence of postoperative complications, physical function changes, and quality of life (QOL) from the perioperative to postdischarge stages in patients with gastrointestinal cancer.

Methods: The study included 97 perioperative patients with gastrointestinal cancer [54 men and 43 women aged $62.5 \pm 12.1$ years (mean \pm SD)] and with or without sarcopenia. Muscle mass was evaluated by the cross-sectional area of skeletal muscle on abdominal computed tomography images, muscle strength by isometric knee extension force, and physical performance by 6 -minute walk distance, and patients were classified into three groups. The incidence of postoperative complications, physical function changes, and QOL were compared among the three groups from the preoperative to postdischarge stages.

Results: The incidence of postoperative complications was significantly greater among patients with sarcopenia compared with the other groups, and QOL in these patients was significantly diminished. In addition, a trend toward decreased physical functioning after surgery was observed in patients with sarcopenia.

Conclusions: Our findings indicate that perioperative sarcopenia influences the incidence of postoperative complications, physical function changes, and QOL after surgery in patients with gastrointestinal cancer.

Key Words: Gastrointestinal cancer patients, Sarcopenia factors, Postoperative course 


\title{
Effect of Exercise Combined with Branched Chain Amino Acid Supplementation on Muscle Strength and Balance Function in Elderly Japanese in Need of Care: A Randomized Controlled Trial
}

\author{
Takashi IKEDA, PT, MS, Tetsuya JINNO, MD, PhD, Sadao MORITA, MD, PhD \\ Tokyo Medical and Dental University, Graduate School of Medical and Dental Sciences \\ Takashi IKEDA, PT, MS, Hiroyuki KUGOTA, PT, Yusuke KUROKI, OT, Kuniko ISHIDA, MD \\ Rehabil-care Shonan Kamakura \\ Hiroshi NAGASAWA, PT, PhD, Ikuko GOMI, RD, PhD \\ Kanagawa University of Human Services, Graduate Program in Human Services \\ Junya AIZAWA, PT, PhD \\ Tokyo Medical and Dental University, Clinical Center for Sports Medicine \& Sports Dentistry \\ Tadashi MASUDA, PhD \\ Fukushima University, Faculty of Symbiotic System Science
}

Purpose: This study examined the effects of exercise combined with branched chain amino acid (BCAA) supplementation on physical function in elderly Japanese in need of care.

Materials and methods: This randomized controlled study enrolled 52 participants. Participants were randomly divided into the following two groups: a BCAA group $(n=27)$ that ingested $6 \mathrm{~g}$ of BCAA supplement 10 min before starting exercise, and a control group $(n=25)$ that ingested $6 \mathrm{~g}$ of maltodextrin. Exercises involved muscle strength exercises requiring $30 \%$ of voluntary maximum contraction, $10 \mathrm{~min}$ of aerobic exercise, and $15 \mathrm{~min}$ of balance function exercise. The 52 participants were followed for 3 months. We determined upper and lower limb isometric strength, grip strength, timed up-and-go test, functional reach distance (FRT), Frenchay Activities Index, and number of rehabilitation sessions. Evaluations were conducted before starting and after 3 months of supplementation.

Results: The mean number of rehabilitation sessions was 18.8 for the BCAA group and 16.1 for the control group. The two factor analysis of variance showed that FRT was significantly differed in nutrition factor at the BCAA group after 3 months of supplementation. Other factors did not differed between two groups after 3 months.

Conclusion: This study found that rehabilitation combined with BCAA supplementation improved FRT. This result suggests that one or two rehabilitation session each week combined with BCAA supplementation may improve balance function.

Key Words: Branched chain amino acid, Physical function, Day care with rehabilitation service 


\title{
Evaluation of THE GROCERY SHELVING TASK as the Assessment of the Motor Function of Upper-limbs in Patients with COPD
}

\author{
Mizuki SATO, PT \\ JA Omagari Kosei Medical Center \\ Azusa ITO, PT \\ Yamamoto Kumiai General Hospital \\ Masahiro IWAKURA, PT, Atsuyoshi KAWAGOSHI, PT, PhD \\ Akita City General Hospital \\ Yoshino TERUI, PT, MS \\ Akita Prefectural Center for Rehabilitation and Psychiatric Medicine \\ Mizuki SATO, PT, Masahiro IWAKURA, PT, Yoshino TERUI, PT, MS, Masahiro SATAKE, PT, PhD, \\ Takanobu SHIOYA, MD, PhD \\ Akita University Graduate School of Health Sciences
}

Purpose: This study's primary purpose was to verify the relationship between the cardiopulmonary response on a grocery shelving task (GST) and the motor function of the upper limbs in patients with chronic obstructive pulmonary disease (COPD). The secondary purpose was to evaluate the usefulness of the GST in the assessment of these patients' upper-limbs motor function.

Methods: We measured the respiratory gas exchange, heart rate and blood pressure and evaluated the modified Borg scale scores during both the GST and a UIULXT (unsupported incremental upper limb exercise test) in 10 COPD patients (mean age $75 \pm 9 \mathrm{yrs}$ ) and 10 healthy elderly subjects (69 $\pm 10 \mathrm{yrs)}$. We analyzed the relationships among the GST performance and respiratory function, respiratory muscle strength, grip strength, PFSDQ-M (pulmonary functional status and dyspnea questionnairemodified) score, m-MRC (the modified medical research council scale), and physical activity of the upper limbs and the whole body.

Result: The exercise intensity achieved by the patients on the GST was $2.3 \pm 1.1$ METs. Their values of $\dot{\mathrm{V}} \mathrm{CO}_{2} / \mathrm{kg}$, systolic blood pressure, $\mathrm{SpO}_{2}$, and modified Borg scale were significantly lower than their UIULXT scores. The time needed to complete the GST (GST time) was $44.1 \pm 10.7 \mathrm{~s}$ in the COPD patients and $38.9 \pm 3.7 \mathrm{~s}$ in the healthy elderly subjects. The GST time, grip strength and physical activity of the upper limbs were positively correlated, whereas the GST time and the values of PFSDQM and m-MRC were negatively correlated.

Conclusion: Our results suggest that the GST is a relatively small-load assessment test for COPD patients and that the GST could be used to accurately evaluate the dyspnea, fatigue and physical activity of the upper limbs in COPD patients' activities of daily living.

Key Words: GST, UIULXT, COPD, The motor function of upper-limbs 


\title{
Different Effects of Motor Learning between Visual and Auditory Feedback Exercises in Dynamic Postural Balance
}

\author{
Naoya HASEGAWA, PT, MS, Hiroki MANI, PT, PhD, Kenta TAKEDA, PT, MS, Moe SAKUMA, PT \\ Graduate School of Health Science, Hokkaido University
}

Naoya HASEGAWA, PT, MS

Department of Rehabilitation, Sapporo Yamanoue Hospital

Satoshi KASAHARA, PT, PhD, Tadayoshi ASAKA, PT, PhD

Department of Rehabilitation Science, Faculty of Health Sciences, Hokkaido University

Purpose: The purpose of this study was to compare the learning effects between visual and auditory feedback exercises which required voluntary weight shifts in dynamic postural balance.

Methods: Twenty healthy young adults participated in this study. The subjects were equally divided into two groups randomly. The subject was required to match his or her center of pressure (COP) to visual targets, which were shown in a monitor, in the forward-backward direction at three test sessions (pre-practice, post-practice, and retention). The subjects were received an additional auditory feedback (AF) or visual feedback (VF) in conjunction with the displacements of COP during the practice session. The root mean square of the distances from the target to the COP (RMS) was calculated to determine the accuracy of the weight shifts.

Results: The mean RMS at the post-practice session was reduced significantly compared to that at the pre-practice session in each group. The reduction in the mean RMS at the retention session was constant in the AF group. On the other hand, the mean RMS at the retention session was increased compared to that at the post-practice session in the VF group.

Conclusion: In dynamic postural balance using the intermittent sensory feedback practices, AF is superior to the VF for the motor learning.

Key Words: Motor learning, Sensory feedback, Dynamic balance 


\title{
The Relation of the Two-Square Step Test to Activities of Daily Living and Walking Ability in Elderly Hospitalized Patients
}

\author{
Shingo KOYAMA, PT, Yuji MORIO, PT, MSc, Hironobu KATATA, PT, Hiroyuki SHIMIZU, MD, PhD \\ St Marianna University School of Medicine Hospital \\ Kazuhiro IZAWA, PT, PhD \\ Kobe University \\ Daisuke ISHIYAMA, PT \\ Kawasaki Municipal Tama Hospital \\ Maiko YAGI, PT, PhD \\ St Marianna University School of Medicine Toyoko Hospital, Kawasaki
}

Purpose: To test the reproducibility and efficacy of the Two-Square Step Test (TSST) in relation to activities of daily living (ADLs) and the ability to walk independently, and to determine the cutoff score for independently walking patients.

Methods: Elderly hospitalized patients 65 years and over were studied. The reproducibility of the TSST was studied in individual patients and between patients. Then, to determine the efficacy of the TSST, we compared it with each exercise of the Four-Square Step Test (FSST) and the Functional Independence Measure (FIM) and determined the cutoff score for independent walking.

Results: The correlation coefficient among individual patients and between these patients and the group of tested patients for the TSST was 0.98 . There was significant correlation between the TSST and the FSST $\left(r_{\mathrm{s}}=0.82\right)$ and between the TSST and the FIM $\left(r_{\mathrm{s}}=0.73\right)$. The cutoff for TSST score to determine the ability to walk independently was 25.5 .

Conclusion: Both the reproducibility and efficacy of the TSST were excellent. We also investigated the correlation between ADLs and independent walking ability. Because the cutoff score for independent walking ability was clarified, we concluded that the TSST was applicable for clinical use.

Key Words: Balance Test, Walking Ability, Activities of Daily Living 


\title{
The Effect of Inhibitory Factors on 100-m Independent Ambulation Immediately after Cardiovascular Surgery in Patients Aged $\geq 80$ Years
}

\author{
Takeshi SHIBUKAWA, PT \\ Shiga University of Medical Science Hospital \\ Kenta KAMISAKA, PT, MSc \\ Kitano Hospital, The Tazuke Kofukai Medical Research Institute \\ Satoshi YUGUCHI, PT, PhD \\ The Sakakibara Heart Institute of Okayama \\ Masayuki TAHARA, PT \\ Higashi Takarazuka Satoh Hospital \\ Keisuke OURA, PT, MSc \\ Fukuyama Cardiovascular Hospital \\ Michitaka KATO, PT, MSc \\ Shizuoka Medical Center \\ Yusuke HANAFUSA, PT, PhD \\ Saitama Medical University International Medical Center \\ Masakazu SAITOH, PT, PhD \\ Sakakibara heart Institute \\ Koji SAKURADA, PT, MSc \\ The Cardiovascular Institute \\ Tomoyuki MORISAWA, PT, MSc \\ Hyogo University Health Sciences \\ Tetsuya TAKAHASHI, PT, PhD \\ Tokyo University of Technology
}

Purpose: This study aimed to identify factors associated with inhibition of independent ambulation and progression of rehabilitation after cardiovascular surgery in patients aged $\geq 80$ years.

Methods: This study involved 557 consecutive patients who underwent cardiovascular surgery (male: 278; mean age: 83.3 years) at 12 facilities in Japan. The patients were classified into 4 groups on the basis of the first postoperative day (POD) of 100-m independent ambulation: (1) early independence group (POD: $\leq 5)$, (2) favorable group (POD: 6-8), (3) delay group (POD: $\geq 9$ ), and (4) non-independence group, and were assessed.

Results: The number of patients in each group was as follows: early independence group, 271 (48.6\%); favorable group, 104 (18.7\%); delay group, 90 (16.2\%); and non-independence group, 92 (16.5\%). In all groups except the non-independence group, the mean time interval for independent ambulation was 7.0 days. Low ADL before cardiovascular surgery was the most important reason for delayed recovery and non-independence (32.4\%). Multiple logistic analyses revealed that chronic kidney disease was significantly associated with delayed recovery.

Conclusion: Even among very old patients, approximately $70 \%$ regained independent ambulation after cardiovascular surgery. Chronic kidney disease was a leading predictor of delayed recovery of physical activity after cardiovascular surgery.

Key Words: Patients aged $\geq 80$ years, After cardiovascular surgery, 100-m independent ambulation 


\title{
Short-term Change and Its Predictors in Life-space: A Small Multicenter Study among Non-active Older Outpatients in Rehabilitation Facilities
}

\author{
Shinya MORIKAWA, PT, MS, Chiaki TANIGUCHI, PT \\ Hohsyasen Daiichi Hospital \\ Kotaro TAMARI, PT, PhD \\ Instituto Nacional de Rehabilitación, Perú \\ Keizo TOKUMARU, PT, PhD \\ Bukkyo University School of Health Science
}

Purpose: The objectives of the current study were to investigate predictors of a short-term change in life-space, and to determine the diagnostic characteristics of the model among older outpatients in rehabilitation facilities.

Method: A total of 82 subjects aged 60 or older took part in this study. A poor outcome was defined as deterioration or a constant low state in life-space, determined by life-space assessment measured at baseline and at a 5-month follow-up, respectively. Independent variables were a series of measurements, including physical and psychosocial functioning, comorbidities, exercise capacity, and demographic factors. A hierarchical binominal logistic regression analysis was carried out to identify independent predictors, with age, sex, body mass index, previous intervention period before baseline, and number of individual therapies entered as confounders. Finally, diagnostic properties were computed using a life-space change prediction model.

Results: A low self-efficacy and grip strength were significant predictors of short-term deterioration/ constant low state in life-space among the studied population. The sensitivity of this model was $82.6 \%$, specificity $72.2 \%$, positive likelihood ratio 2.97 , and negative likelihood ratio 0.24 .

Conclusion: The results indicate that grip strength and self-efficacy predict a short-term change in life-space among non-active older out-patients in rehabilitation facilities.

Key Words: Life-space assessment, Life-space, Diagnostic characteristics, Predictors, Non-active 


\title{
Difference in In-hospital Postoperative Rehabilitation Outcome in Patients with Thoracic and Thoracoabdominal Aortic Aneurysms
}

\author{
Yuichi ADACHI, PT \\ Sakakibara Heart Institute \\ Kenta KAMISAKA, PT, MSc \\ Kitano Hospital, The Tazuke Kofukai Medical Research Institute \\ Masayuki TAHARA, PT \\ Higashi Takarazuka Satoh Hospital \\ Keisuke OURA, PT, MSc \\ Fukuyama Cardiovascular Hospital \\ Takeshi SHIBUKAWA, PT \\ Shiga University of Medical Science Hospital \\ Yusuke HANAFUSA, PT, PhD \\ Saitama Medical University International Medical Center \\ Satoshi YUGUCHI, PT, PhD \\ The Sakakibara Heart Institute of Okayama \\ Michitaka KATO, PT, MSc \\ Shizuoka Medical Center \\ Koji SAKURADA, PT, MSc \\ The Cardiovascular Institute \\ Tetsuya TAKAHASHI, PT, PhD \\ Tokyo University of Technology
}

Purpose: The aim of this study was to evaluate the outcome of postoperative rehabilitation in patients who underwent repair for thoracic and thoracoabdominal aortic aneurysms.

Method: Two-hundred and four patients (153 male, 51 female, aged $68 \pm 13$ years) were divided into 3 groups by the site of aortic repair: group A (ascending aorta repair), group B (aortic arch repair), and group C (descending and thoracoabdominal aorta repair). In each group, we categorized the patients into 3 grades by postoperative days to achieve 100-m unassisted walk; early ( $\leq 4$ days), intermediate (5-8 days), and delayed ( $\geq 9$ days) or not achieved. Based on this category, we assessed the clinical characteristics, perioperative data, progression of postoperative rehabilitation, and also the reasons for delayed or not achieved.

Result: There were more patients graded as early in group A, and more patients graded as delayed or not achieved in groups B and $\mathrm{C}(\mathrm{p}<0.05)$. The main reason for delayed or not achieved was cerebral vascular disease in group B, and spinal cord infarction in group $\mathrm{C}(\mathrm{p}<0.05)$.

Conclusion: The progression of postoperative rehabilitation tended to be delayed or unable to achieve unassisted 100-m walk in patients who underwent aortic arch repair and descending and thoracoabdominal aorta repair, and the main reason was perioperative complication of cerebral vascular disease in the former and spinal cord infarction in the latter.

Key Words: Thoracic aortic aneurysm, Thoracoabdominal aortic aneurysm, Postoperative rehabilitation 


\title{
Factors Associated with Maintenance of Physical Activity among Older Adults Who Participated in the Class in a Care Prevention Program
}

\author{
Satoko NAKANO, PT, MS \\ Graduate School of Comprehensive Human Sciences, University of Tsukuba \\ Junko OKUNO, PhD, Takako FUKASAKU, PhD, Hisako YANAGI, MD, PhD \\ Faculty of Medicine, University of Tsukuba \\ Kazushi HOTTA, OT, PhD \\ Ibaraki Prefectural University of Health Sciences \\ Noriko YABUSHITA, PhD, Miyuki NEMOTO, PhD, Kiyoji TANAKA, PhD \\ Faculty of Health and Sport Sciences, University of Tsukuba
}

Purpose: The present study was conducted to identify the determinants of maintenance of physical activity among older adults who participated in a care prevention program.

Methods: The subjects were 309 older adults who participated in a care prevention program. The subjects were divided into three groups, namely the exercise continuation group, unstable exercise continuation group, and non-exercise group, by using an action transformation stage about the exercise. We investigated the baseline characteristics, social participation, body function, and psychological factors of the subjects by administering a self-reported questionnaire and conducting physical performance tests.

Results: Social participation and functional factors were related to exercise activity. Multiple logistic regression analysis revealed that a lifestyle with exercise and recovery self-efficacy were associated with maintenance of physical activity.

Conclusions: In this study, we demonstrated that incorporating exercise into one's lifestyle and enhancement of recovery self-efficacy are important factors that promote physical activity among older adults who participate in a care prevention program.

Key Words: Care prevention, Maintenance of physical activity, Action planning, Recovery Self-Efficacy 


\title{
The Combined Effect of Wheelchair Propulsion Practice and Rhythmic Auditory Stimulation on the Short-stroke Wheelchair Propulsion of Parkinson's Disease Patients: A Report on 3 Cases
}

\author{
Tatsuro OKAMURA, PT \\ Department of Rehabilitation, Ojiya Sakura Hospital \\ Ryousaku KOBAYASHI, PT, PhD \\ Department of Physical Therapy, Niigata University of Health and Welfare
}

Purpose: In this study, we aimed to investigate whether wheelchair propulsion by patients with severe Parkinson's disease can be improved by using rhythmic auditory stimulation.

Methods: Three wheelchair-bound patients (subjects A, B, and C) with stage 4 Parkinson's disease, as assessed using the Hoehn \& Yahr scale, were included in this study. The items assessed were the time required to propel the wheelchair $10 \mathrm{~m}$ at full exertion and the number of strokes. Each trial was divided into the following 4 phases: baseline, intervention, after intervention, and followup. Measurements were performed without rhythmic auditory stimulation during the baseline and follow-up, but with rhythmic auditory stimulation during and after the intervention. The intervention comprised 5 minutes of wheelchair propulsion, which was practiced twice per week for 4 weeks.

Results: The time required for wheelchair propulsion and the number of strokes were markedly decreased after the intervention compared to that at baseline for subjects A (required time: $31 \%$, number of strokes: $28 \%$ in comparison to $100 \%$ at baseline), B $(37 \%, 26 \%)$, and C $(54 \%, 65 \%)$. The effect was continued into the follow-up phase for subjects A and B, but not for subject C.

Conclusion: This study suggests the possibility that wheelchair propulsion practice combined with rhythmic auditory stimulation is effective in improving wheelchair propulsion by patients with Parkinson's disease.

Key Words: Parkinson's disease, Wheelchair propulsion, Rhythmic auditory stimulation 


\title{
MRI Analysis of the Range of Motion of Spinal Segments and the Sacroiliac Joint in the Sagittal Plane in the Prone Position with Trunk Extension
}

\author{
Masafumi HATA, PT, MS, Kazuna ICHIKAWA, PT, MS \\ Department of Physical Therapy, Senkawa-Shinoda Orthopedic Clinic \\ Masafumi HATA, PT, MS, Kazuna ICHIKAWA, PT, MS, Sho MITOMO, PT, MS, Daisuke OGAWA, PT, MS \\ Doctoral Course, Deparment of Physical Therapy, Graduate School of Human Health Sciences, Tokyo \\ Metropolitan University \\ Hideyuki USA, PT, PhD \\ Division of Physical Therapy, Faculty of Health Sciences, Tokyo Metropolitan University \\ Sho MITOMO, PT, MS \\ Department of Physical Therapy, Kawakita General Hospital \\ Daisuke OGAWA, PT, MS \\ Department of Physical Therapy, Faculty of Health Sciences, Mejiro University \\ Masashi MATSUMURA, PT, PhD \\ Department of Physical Therapy, Faculty of Health Sciences, Kyorin University \\ Atsushi SENOO, RT, PhD \\ Deparment of Radiological Sciences, Graduate School of Human Health Sciences, Tokyo Metropolitan \\ University \\ Hitoshi TAKEI, PT, PhD \\ Deparment of Physical Therapy, Graduate School of Human Health Sciences, Tokyo Metropolitan University
}

Purpose: The purpose of this study was to analyze the range of motion of spinal segments and the sacroiliac joint in the sagittal plane when the trunk moves from the prone to the extension position.

Methods: Thirteen asymptomatic female volunteers participated in this study. The prone position with no trunk extension (prone), slight trunk extension, and moderate trunk extension (referred to as the puppy position) were analyzed using magnetic resonance imaging (MRI). The range of motion of the lower thoracic and lumbar zygapophysial joints (from Th11/12 to L4/5), the lumbosacral joint (L5/ S1), and the sacroiliac joint was investigated.

Results: In the slight trunk extension position, Th12/L1 to L3/4 showed more extension than did L4/5 and L5/S1. In the puppy position, Th12/L1 to L4/5 showed more extension than did L5/ S1. Moreover, a larger angle was measured from Th12/L1 to L4/5 in the puppy position than was measured in the slight trunk extension position. There was almost no movement of the sacroiliac joint in either position.

Conclusions: This study showed that the primary segments showing extension in the slight trunk extension position were Th12/L1 to L3/4, while those that showed extension in the puppy position were Th12/L1 to L4/5. In addition, Th12/L1 to L4/5 was more extended in the puppy position than in the slight trunk extension position. The sacroiliac joint showed almost no movement in either position.

Key Words: Trunk extension position, Range of motion, Magnetic resonance imaging 


\title{
Effect of Expiratory Breathing Load on Trunk Muscle Activities During Open Kinetic Chain Exercise
}

\author{
Miyuki KOMATSU, PT, MS, Akira TAMAKI, PT, PhD \\ Department of Rehabilitation Science, Graduate School of Health Science, Hyogo University of Health \\ Sciences \\ Kotatsu NAGAI, PT, PhD, Masami HIDAKA, PT, PhD, Kotaro KAWAGUCHI, PT, PhD \\ Department of Physical Therapy, School of Rehabilitation, Hyogo University of Health Sciences
}

Purpose: To investigate trunk muscle activities during open kinetic chain (OKC) exercise with forced expiration.

Methods: Twenty-five healthy young women were recruited for this study. We measured the muscle thickness of the transverse abdominis and the muscle activities of other abdominal muscles (rectus abdominis, external oblique, and internal oblique) during OKC exercise by using ultrasonography and electromyography (EMG), respectively. OKC exercise included the following four grades of forced expiration: quiet breathing, 10\% maximal static expiratory pressure (PEmax), 20\% PEmax, and 30\% PEmax.

Results: The thickness of the transverse abdominis was markedly higher during exercises with $10 \%$, $20 \%$, and 30\% PEmax than that during exercises with quiet breathing. In addition, 20\% PEmax also showed to be more significant than 30\% PEmax. EMGs of three abdominal muscles during the tasks with forced expiration were higher than that of the task in quiet breathing, though there was no significant difference in EMGs during 10\% PEmax, 20\% PEmax, and 30\% PEmax.

Conclusion: OKC exercise with forced expiration at 20\% PEmax or less could increase the muscle activities of the transverse abdominis as well as other trunk muscles.

Key Words: Open kinetic chain exercise, Transversus abdominis muscle, Load strength of the expiration 


\section{Usefulness of the Stroke Impairment Assessment Set in Stroke Patients: Standardized Response Mean and Minimal Clinically Important Difference}

Ken-ichi KUMAGAE, PT, Kota YAMAUCHI, PT, Yuki KOBAYASHI, PT, Risa HAGIWARA, PT, Kimi IWAMATSU, PT, Yasuhiro KOYANAGI, PT, MS, Shigeru FUJIMOTO, MD, PhD, Satoshi SUZUKI, MD, PhD

Steel Memorial Yawata Hospital

Satoshi SUZUKI, MD, PhD

Fukuoka Kieikai Hospital, Stroke Center

Purpose: This study aimed to determine the standardized response mean (SRM) and the minimal clinically important difference (MCID) for the Stroke Impairment Assessment Set (SIAS).

Methods: The participants in this cohort study were 244 patients who were admitted to hospital and received rehabilitation because of stroke. Participants were assessed by the SIAS on the 7th and 21st day after admission, and responsiveness was assessed using the SRM. Improvement in ambulatory function was used to detect a clinically meaningful change. The MCID for the SIAS was estimated by the meaningful change.

Results: The SRM and the MCID for the SIAS were estimated as 0.61 (95\% CI, 0.45-0.74) and an improvement in the score of 2 , respectively.

Conclusions: Responsiveness of the SIAS is moderate. Improvement in the SIAS score of 2 is related to the improvement of ambulatory function. Therefore, the SIAS is a useful assessment tool for stroke.

Key Words: Minimal Clinically Important Difference, Standardized Response Mean, Stroke Impairment Assessment Set 


\section{An Examination of Factors Associated with the Discharge Home in Acute Ischemic Stroke Patients}

Yota KUNIEDA, PT, MS, Hiroshi MIKI, PT, MS, Tomoya IMAI, PT, MS, Haruhiko HOSHINO, MD

Tokyo Saiseikai Central Hospital

Osamu NITTA, PT, PhD

Tokyo Metropolitan University

Purpose: The aim of this study was to examine predictive factors connected with the discharge destination in acute ischemic stroke patients.

Method: 58 patients were assigned either to the discharged home group and the changing hospital group. We retrospectively studied the factors from the medical records and compared between the two groups. Next, we analyzed the significantly differenced factors with multiple logistic regression analysis.

Results: The identified significant factors associated with discharge home were sex, the serum albumin(Alb), the total serum protein, the national institute of health stroke scale, the food intake level scale, and existence of higher brain dysfunction. The factors associated with discharge home by the multiple logistic regression analysis used 5 items mentioned above indicated higher brain dysfunction ( $\mathrm{p}=0.000$, odds ratio $0.076,95 \%$ confidence interval 0.018-0.312), and Alb ( $\mathrm{p}=0.021$, odds ratio 0.178, 95\% confidence interval 0.041-0.768). From the results of ROC curves, cut off values for determining discharge direction were $3.95 \mathrm{~g} /$ dlby Alb.

Conclusion: The levels of Alb and higher brain dysfunction at the time of hospitalization might be the factors for discharging home with acute ischemic stroke patients.

Key Words: Acute ischemic stroke, Discharge home, Factors 


\section{Immediate Beneficial Effects of Action-observation, and Its Effects Underlying Working Memory}

Tsubasa KAWASAKI, PT, PhD, Hidefumi ARAMAKI, PT, MSc, Ryosuke TOZAWA, PT, MSc, Munenori KATO, PT, PhD

Ryotokuji University

Purpose: To investigate whether action observation had immediate effects on hand coordination and whether its effects were correlated with working memory function, using the Trail Making Test (TMT) part B.

Methods: Thirty-one adults participated in this study. Of them, 20 participants watched a video of an expert performing a ball-rotation task using his left hand for one minute. The participants' ballrotation performance using the left hand was measured by recording the time required to complete 10 rotations and the number of times the ball was dropped in the pre- and post-observation sessions. In the control group, 11 participants performed the ball-rotation task without watching any video. To assess the working memory function, the time required to complete the TMT part A and B was recorded.

Results: Improvement ratios were significantly higher in the observation group than in the control group. In addition, there were significant correlations between the effects of observational learning in the post-observation sessions and the time required to complete TMT part B and $\Delta$ TMT (i.e., the time required to complete part $\mathrm{B}$ minus part $\mathrm{A}$ ).

Discussion: These findings suggest that action observation immediately encouraged an improvement in hand coordination, and its beneficial effects were related to working memory function. In conclusion, action observation combined with interventions to enhance working memory function may immediately encourage the improvement of motor skills.

Key Words: Observational learning, Immediate effects, Working memory 


\section{Effects of Spinal Deformities on Mechanical Energy Flow between Left and Right Legs During Gait}

Toru SAKUMA, PT, MS

Tsukuba University of Technology

Michiyoshi AE, PhD

University of Tsukuba

Yasuto KOBAYASHI, MS

Ibaraki Prefectural University of Health Sciences

Purpose: The study investigated the effect of spinal deformities on mechanical energy flow between the left and right legs during gait.

Methods: Five spondylosis patients and nine healthy subjects were asked to walk. Gait motion was captured with a VICON MX T-series camera and ground reaction forces during stance phase were collected with two Kistler force platforms.

Results: Pelvic angular velocity at early swing phase and energy flow (of joint force flowing from the trunk to the thigh) at the hip were lower in the patients than in the healthy subjects. Furthermore, energy transfer between segments was lower in patients than in healthy subjects.

Conclusions: Results suggested that increasing the pelvic angular velocity by leading the hip joint of the recovery leg forward and upward at early swing phase appears to be important for the effective use of mechanical energy in spondylosis patients during gait.

Key Words: Gait, Spinal deformity, Mechanical energy flow 


\section{Physical and Psychological Characteristics Related to Improvement from a Frail Condition among of Elderly Individuals after 2-years Ago in an Urban Area}

Takeshi KERA, PT, PhD, Hisashi KAWAI, PhD, Hideyo YOSHIDA, MD, PhD, Hirohiko HIRANO, DMD, PhD, Yoshinori FUJIWARA, MD, PhD, Shuichi OBUCHI, PT, PhD

Tokyo Metropolitan Geriatric Hospital and Institute of Gerontology

Takeshi KERA, PT, PhD

Fuke Hospital

Motonaga KOJIMA, PT, CSW, PhD

University of Tokyo Health Sciences

Kazushige IHARA, MD, $\mathrm{PhD}$

Toho University School of Medicine

Purpose: Because Frail is factor of healthy life expectancy and vital prognosis, prevent and improving of frail are important. The aim of our study was to reveal the factor of improving from frail.

Methods: Subjects included 98 frail elderly persons in the urban area. They were divided into improve group $(n=38)$ from frail and stable group $(n=40)$ by physical and social evaluation after 2-year, and both group were compared. Furthermore, Logistic regression analysis examined variables predicting frailty at 2-year follow-up.

Results: The motor functions were higher and depression score, fall score and houseboundness in the improved group was lower than in the stable group. The grip power and score of physical and life function in baseline were included as predictor of improving from fail.

Conclusion: We concluded that increasing physical function on daily was needed to improve from frail.

Key Words: Frailty, Predictor, Physical function 